Editorial

\title{
Foreword: 2018 International Symposium on Rail Infrastructure Systems Engineering (i-RISE 2018) ${ }^{\dagger}$
}

\author{
Milan Omasta ${ }^{1, *}$ and Sakdirat Kaewunruen ${ }^{2}$ \\ 1 Faculty of Mechanical Engineering, Brno University of Technology, Antonínská 548/1, 60190 Brno, \\ Czech Republic \\ 2 Laboratory for Track Engineering and Operations for Future Uncertainties (TOFU Lab), \\ School of Engineering, The University of Birmingham, B15 2TT Birmingham, UK; \\ s.kaewunruen@bham.ac.uk \\ * Correspondence: omasta@fme.vutbr.cz; Tel.: +420-54114-3238 \\ + Presented at 2018 International Symposium on Rail Infrastructure Systems Engineering (i-RISE 2018), \\ Brno, Czech Republic, 5 June 2018. \\ Published: 31 August 2018
}

Social and economic growth, security and sustainability around the globe are at risk of being compromised due to aging and failing railway infrastructure systems. This partly reflects a recognised skill shortage in railway infrastructure engineering. This international symposium has thus been established to cross-disciplinary develop practical and scientific skills for the next generation of capable railway engineers. The symposium is part of a project, RISEN, aims to enhance knowledge creation and transfer using both international and intersectoral secondment mechanisms among European Advanced Rail Research Universities/SMEs and Non-EU, world-class rail universities including the University of Illinois at Urbana Champaign (USA), Massachusetts Institute of Technology (USA), University of California Berkeley (USA), Southwest Jiaotong University (China), Tsinghua University (China), University of Wollongong (Australia), Railway Technical Research Institute (Japan), University of Sao Paolo (Brazil), and Iranian University of Science and Technology (Iran). The European rail universities include the University of Birmingham (UK), KTH Royal Institute of Technology (Sweden), Polytechnic University of Milan (Italy), Tampere University of Technology (Finland), University of Porto (Portugal), Norwegian University of Science and Technology (Norway), Brno University of Technology (Czech), Delft University of Technology (The Netherlands), and Polytechnic University of Valencia (Spain). Industry partners are MerMec (France), RoadScanners (Finland), Evoleo Technologies (Portugal) and Evoleo GmbH (Germany). In addition, the key sponsors are China Academy of Railway Sciences (China), Japan Society for the Promotion of Science (Japan) and EU COST Actions. This project adds research skill mobility and innovation dimension to existing bilateral collaborations between universities through research exchange, joint research supervision, summer courses, international training and workshops, and joint development of innovative inventions. It spans over 4 years from April 2016 to March 2020. The authors are therefore sincerely grateful to the European Commission for the financial sponsorship of the H2020-RISE Project No. 691135 "RISEN: Rail Infrastructure Systems Engineering Network", which enables a global research network that tackles the grand challenge of railway infrastructure resilience and advanced sensing in extreme environments (www.risen2rail.eu).

RISEN aims to produce the next generation of engineers and scientists needed to meet the challenge of providing sustainable, smart and resilient railway infrastructure systems critical for maintaining European competitiveness. The emphasis will be placed on the resilience and adaptation of railway and urban transport infrastructures using integrated smart systems. Such critical areas of the research theme will thus be synergised to improve response and resilience of rail infrastructure systems to climate change, extreme events from natural and human-made hazards, and future 
operational demands. In addition, researchers will benefit from the co-location of engineering education, training and research alongside world-class scientists and industry users through this initiative. Lessons learnt from rail infrastructure management will be shared and utilised to assure integrated and sustainable rail transport planning for future cities and communities.

Emerging risks and their significant consequences with no sign of early warning can be evidenced recently by many extreme events such as Nepal Earthquakes, Madrid train bombing, Brussels Metro Attack, etc. Much past research has emphasised the applications of technology in solving front-line problems in the railway industry. Although the practical knowledge has been developed alongside with corporate knowledge, its science and technology are still insufficient in order to innovate and revolutionise the railway industry from the fundamental principle viewpoint. Many fundamental issues, such as choice of materials, durability, capacity, engineering properties, functionality requirements and design concepts, remain unchanged. Together with high turnover rate of technical staff within rail industry worldwide, many incidents have repeated themselves, causing high maintenance costs, service cancellations and delays, and even human lives due to catastrophic incidents. This international symposium has addressed some of the most essential issues currently affecting the safety, reliability and functionality of global rail infrastructure systems. Rebuilding and enhancing urban rail infrastructure faces problems beyond the search for engineering solutions. These issues resonate with one of the major grand challenges engineering in order to restore and improve urban infrastructure and built environments, of which it will not only be necessary to devise new approaches and methods, but to communicate their value and worthiness to society at large. This symposium has unveiled novel and original research topics related to railway infrastructure systems, to which the untrained eyes might not see the difference. Significant impact of the associated research and huge advancements in the rail infrastructure technology and the performance of modern railway infrastructure have been highlighted in the symposium.

The work presented in the symposium has already led to practical improvements in the operation of railways; and we are very certain that further developments building on this work by the teams represented here in RISEN and other collaborators will deliver even greater safety, performance and reliability levels of rail infrastructure systems in the future.

\section{A/Professor Milan Omasta and Dr Sakdirat Kaewunruen}

Co-Chair, 2018 International Symposium on Rail Infrastructure Systems Engineering 